\title{
Physical controls on orographic cirrus inhomogeneity
}

\author{
J. E. Kay ${ }^{1}$, M. Baker ${ }^{2}$, and D. Hegg ${ }^{2}$ \\ ${ }^{1}$ National Center for Atmospheric Research, Boulder, CO, USA \\ ${ }^{2}$ Department of Atmospheric Sciences, University of Washington, Seattle, WA, USA
}

Received: 5 March 2007 - Published in Atmos. Chem. Phys. Discuss.: 10 April 2007

Revised: 17 July 2007 - Accepted: 17 July 2007 - Published: 23 July 2007

\begin{abstract}
Optical depth distributions $(\mathrm{P}(\sigma))$ are a useful measure of radiatively important cirrus $(\mathrm{Ci})$ inhomogeneity. Yet, the relationship between $\mathrm{P}(\sigma)$ and underlying cloud physical processes remains unclear. In this study, we investigate the influence of homogeneous and heterogeneous freezing processes, ice particle growth and fallout, and mesoscale vertical velocity fluctuations on $\mathrm{P}(\sigma)$ shape during an orographic $\mathrm{Ci}$ event. We evaluate Lagrangian $\mathrm{Ci}$ evolution along kinematic trajectories from a mesoscale weather model (MM5) using an adiabatic parcel model with binned ice microphysics. Although the presence of ice nuclei increased model cloud cover, our results highlight the importance of homogeneous freezing and mesoscale vertical velocity variability in controlling $\mathrm{Ci} \mathrm{P}(\sigma)$ shape along realistic upper tropospheric trajectories.
\end{abstract}

\section{Introduction}

\subsection{Background}

Cirrus clouds ( $\mathrm{Ci}$ ), layer clouds that are entirely glaciated, are often optically inhomogeneous. Neglecting Ci optical depth $(\sigma)$ inhomogeneity can lead to large biases in computed radiative fluxes (Fu et al., 2000; Carlin et al., 2002). One useful measure of $\mathrm{Ci}$ inhomogeneity is an optical depth distribution $\mathrm{P}(\sigma)$, i.e., the fraction of $\sigma$ occurring at a given $\sigma$. Understanding physical controls on $\mathrm{Ci} \mathrm{P}(\sigma)$ should improve representation of radiative fluxes in weather and climate models.

In general, $\mathrm{Ci} \sigma$ can be approximated as:

$\sigma=2 \pi R_{\text {eff }}^{2} N_{\text {ice }} \Delta Z$

where $R_{\text {eff }}$ is the ice crystal effective radius [m], $N_{i c e}$ is number concentration of ice crystals $\left[\mathrm{m}^{-3}\right]$, and $\Delta Z$ is the $\mathrm{Ci}$ cloud layer thickness [m].

Correspondence to: J. E. Kay

(jenkay@ucar.edu)
From Eq. (1), we find that for a fixed ice $R_{\text {eff }}$ and $\Delta Z, \mathrm{Ci}$ $\sigma$ are determined primarily by $N_{\text {ice }}$. At a fixed ice water content (temperature), $R_{\text {eff }}$ is largely determined by $N_{\text {icee }}$. Therefore, understanding physical controls on $\mathrm{Ci} N_{\text {ice }}$ is a first-step towards understanding physical controls on $\mathrm{Ci} \mathrm{P}(\sigma)$.

Kärcher and Ström (2003) and Hoyle et al. (2005) concluded that homogeneous freezing and small scale variability (frequencies $\left(v\left[\mathrm{~h}^{-1}\right]\right)$ up to $10 \mathrm{~h}^{-1}$ or spatial scales $<11 \mathrm{~km}$ ) in vertical velocity $\left.\left(w\left[\mathrm{~m} \mathrm{~s}^{-1}\right]\right)\right)$ controlled $N_{\text {ice }}$ distributions measured during the INCA and SUCCESS field campaigns. Haag and Kärcher (2004) found that background number concentrations of ice nuclei $\left(N_{I N}\right)$ reduced modeled $N_{\text {ice }}$, but that IN presence did not significantly alter overall $\mathrm{Ci}$ properties and formation locations. Kay (2006) noted that observed $N_{I N}\left(N_{I N}<0.1 \mathrm{~cm}^{-3}\right.$ (DeMott et al., 2003; Rogers et al., 1998)) and homogeneous freezing at weak synopticscale $w\left(w \ll 5 \mathrm{~cm} \mathrm{~s}^{-1}\right.$ (Mace et al., 2001)) could not explain the mean observed $N_{\text {ice }}$ at Lamont, Oklahoma (USA) $\left(\overline{N_{\text {ice }}}=0.1 \mathrm{~cm}^{-3}\right.$, Mace et al., 2001). Taken together, these studies suggest that observed $\mathrm{Ci} N_{\text {ice }}$ can be largely explained by homogeneous freezing occurring at a range of $w$. These studies also imply that heterogeneous freezing alone cannot explain observed Ci $N_{\text {ice }}$.

Kay et al. (2006) (hereafter K06) assessed physical controls on Lagrangian $\mathrm{P}(\sigma)$ along constant lifting trajectories. For a typical range of $w$, temperatures $(T[\mathrm{~K}])$, and $N_{I N}$, $\bar{\sigma}$ and $\mathrm{P}(\sigma)$ shape depended primarily on $w$. The sensitivity of $\bar{\sigma}$ to $w$ resulted for two reasons: 1) As $w$ increased, Ci $N_{\text {ice }}$ increased, $R_{\text {eff }}$ decreased, and the initial $\sigma$ increased (see Eq. 1). 2) As $R_{\text {eff }}$ decreased, fallout timescales ( $\tau_{\text {fallout }}$ [s]) and cloud lifetimes increased. In other words, the $w$ during freezing controlled both the initial $\sigma$ and the $\sigma$ evolution. In contrast, the addition of IN to lifting parcels had a limited influence on modeled $\sigma$. The addition of observed $N_{I N}$ only reduced $\bar{\sigma}$ and modified $\mathrm{P}(\sigma)$ with large $w$. With small $w$, IN had little influence on the calculated $\bar{\sigma}$ and $\mathrm{P}(\sigma)$ because IN quickly fell out of the parcel, and because the $N_{\text {ice }}$ generated

Published by Copernicus Publications on behalf of the European Geosciences Union. 
A.
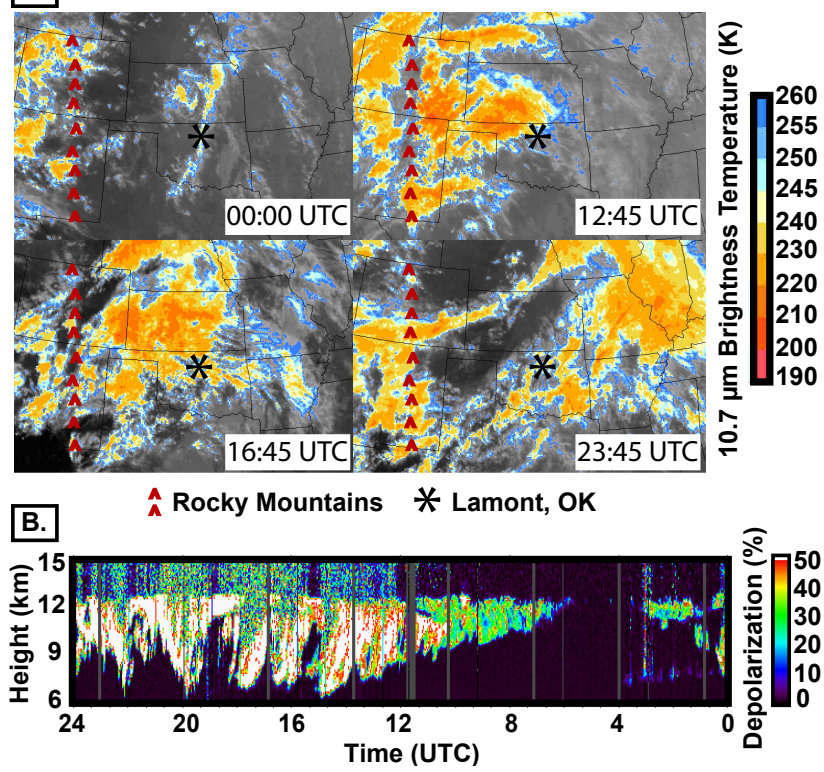

Fig. 1. Infrared satellite and lidar depolarization observations on 19 April 2001: (A) GOES infrared satellite image time series. Low brightness temperatures indicate high cloud tops. (B) Vertically pointing lidar depolarization ratio observations from Lamont. High depolarization ratios $(>10 \%)$ indicate ice.

by homogeneous freezing at low $w$ were comparable to observed $N_{I N}$.

Given the importance of $w$ to $\mathrm{Ci} N_{\text {ice }}$ and $\mathrm{P}(\sigma)$, the influence of realistic $w$ sequences on Ci formation and evolution should be evaluated. Unfortunately, it is difficult to predict air flow and measure $w$ along $\mathrm{Ci}$ evolution pathways. With the exception of wave cloud studies (e.g., INTACC (Field et al., 2001), FIRE II (Heymsfield and Miloshevich, 1995)), there is a dearth of Lagrangian $w$ observations. As a substitute for measuring Lagrangian $w$ in the atmosphere, previous studies (e.g., Hoyle et al., 2005; Haag and Kärcher, 2004) have statistically constructed Lagrangian $w$ trajectories. In these studies, observed distributions of small-scale $w$ were superimposed on Lagrangian displacement trajectories derived from large-scale atmospheric models (horizontal resolution $>40 \mathrm{~km}$ ). Because Lagrangian $w$ measurements are difficult to obtain, and because statistically constructed $w$ trajectories are not necessarily realistic, kinematic trajectories extracted from mesoscale weather models $(4 \mathrm{~km}<$ horizontal resolution $<40 \mathrm{~km}$ ) could serve as a useful proxy for Lagrangian $w$ observations. Mesoscale weather model trajectories capture mesoscale $w$ variability $\left(2 \mathrm{~h}^{-1}<v<10 \mathrm{~h}^{-1}\right)$ and provide a realistic and self-consistent measure of Lagrangian $w$ evolution.

\subsection{Study goals and organization}

In this study, we investigate physical controls on orographic $\mathrm{Ci} \mathrm{P}(\sigma)$ using the $\mathrm{K} 06$ parcel model and $w$ trajectories derived from the PSU/NCAR mesoscale model (MM5) (Grell et al., 1994). We selected an orographic Ci case study because mountainous terrain provides a natural laboratory for investigating the influence of mesoscale $\left(2 \mathrm{~h}^{-1}<v<10 \mathrm{~h}^{-1}\right) w$ variability $\left(w=1-300 \mathrm{~cm} \mathrm{~s}^{-1}\right.$, cooling rates $\left.=1-100 \mathrm{~K} \mathrm{~h}^{-1}\right)$ on $\mathrm{Ci} \mathrm{P}(\sigma)$, and because orographic $\mathrm{Ci}$ are often missed by climate models (Dean et al., 2005). We note that orographic $\mathrm{Ci}$ typically form in environments with larger w than non-orographic $\mathrm{Ci}$.

In Sect. 2, we introduce the orographic $\mathrm{Ci}$ case study using observations. In Sect. 3, we present and evaluate the meteorology and $w$ forecasted by MM5. In Sect. 4, we describe both our methods for estimating $\sigma$ evolution with the K06 parcel model and our trajectory parcel model experiments. Section 5 contains our results: we evaluate the influence of $w$ and IN on $\mathrm{Ci} \mathrm{P}(\sigma)$ calculated along realistic upper tropospheric trajectories. We compare parcel model $\mathrm{Ci}$ to the Ci generated by a standard MM5 bulk microphysics scheme, the Reisner II scheme (Reisner et al., 1998). Their intercomparison is interesting because the Reisner II scheme neglects the influence of $w$ on $\mathrm{Ci} N_{\text {ice }}$. In fact, the $N_{\text {ice }}$ predicted by the Reisner II scheme at $\mathrm{Ci}$ formation temperatures $\left(T<-30^{\circ} \mathrm{C}\right)$ is a constant $N_{\text {ice }}=0.1 \mathrm{~cm}^{-3}$. Finally, we assess which physical factors could explain the observed $\mathrm{Ci}$ formation and broad $\mathrm{P}(\sigma)$. Section 6 contains a summary and discussion of our results.

\section{April 2001 Ci observations}

On 19 April 2001, orographic Ci formation and evolution was observed by the GOES infrared satellite and a vertically pointing Raman lidar located at Lamont, Oklahoma (OK), hereafter Lamont (Fig. 1). From 06:00 to 16:00 UTC, orographic Ci formed in the lee of the Southern Rocky Mountains. The $\mathrm{Ci}$ were then advected East with the upper level winds. Approximately 5 to $6 \mathrm{~h}$ after formation, the $\mathrm{Ci}$ were observed by the lidar above Lamont.

The lidar-observed $\mathrm{Ci}$ had a constant cloud top height of approximately $12.7 \mathrm{~km}$, but a cloud base that varied from 6.5 to $11 \mathrm{~km}$ (Fig. 1). Two independent $\sigma$ retrievals, one based on emissivity shape in the atmospheric window retrieved from Atmospheric Emitted Radiance Interferometer (AERI) observations (Turner, 2005), and the other based on Beer's law and the lidar backscatter below and above cloud, were generally consistent when $\sigma<3$. Retrieved $\mathrm{Ci} \sigma$ increased monotonically from 06:00 to 12:00 UTC and then varied from $\sigma<0.1$ to $\sigma \sim 3$ (Fig. 2). From 12:00 to 24:00 UTC, $\sigma$ variability resulted in a broad $\mathrm{P}(\sigma)$ (Fig. 2). The lidar observations reveal that $\Delta Z$ variations contributed to the observed broad $\mathrm{P}(\sigma)$ (Fig. 2). Although the lidar observations do not 

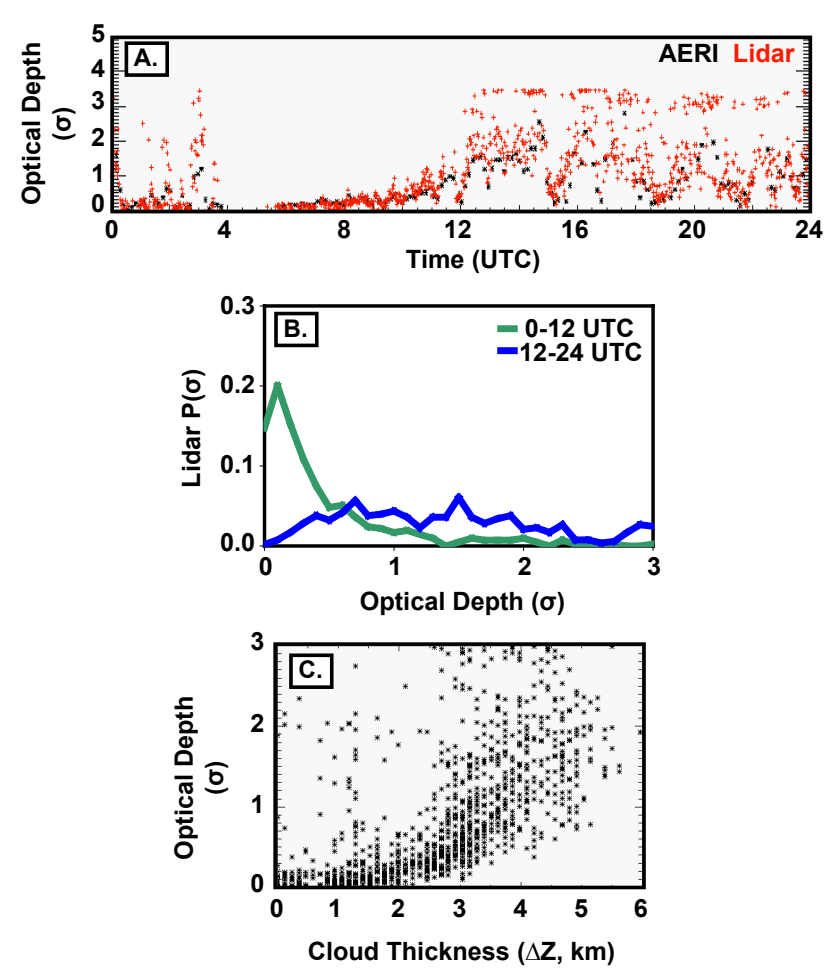

Fig. 2. 19 April $2001 \sigma$ observations: (A) $\sigma$ time series. Time series of $\mathrm{Ci} \sigma$ based on two independent retrieval methods (AERI, lidar). (B) 12-h lidar-derived $\mathrm{P}(\sigma)$. (C) lidar-derived $\sigma$ vs. physical cloud thickness from 08:00 to 24:00 UTC.

reveal the influence of $N_{\text {ice }}$ and $R_{\text {eff }}$ on the observed $\sigma$, the large amount of scatter in the observed relationship between $\sigma$ and $\Delta Z$ shows that the observed $\Delta Z$ variability explains only a small fraction of the observed $\sigma$ variability (Fig. 2).

\section{April 2001 MM5 forecast}

\subsection{MM5 methods}

We ran the MM5 with three nested domains for the 19 April 2001 orographic $\mathrm{Ci}$ event both to forecast the meteorology, and to enable calculation of Lagrangian $w$ trajectories (Fig. 3, Table 1). All MM5 domains included the Front Range of the Rocky Mountains, where the Ci formed, and Lamont, where the Ci passed overhead (Fig. 1).

\subsection{MM5 meteorology}

At 12:00 UTC on 19 April 2001, the MM5 forecasted a broad upper-level ridge over the central United States, a low pressure system developing in Montana, and a trough in the lee of the Rocky Mountains (Fig. 4). Both the developing Montana low and the lee trough contributed to a weak North-South trending warm front. A cross section of equivalent potential temperatures shows the lee trough, a cold front aloft above

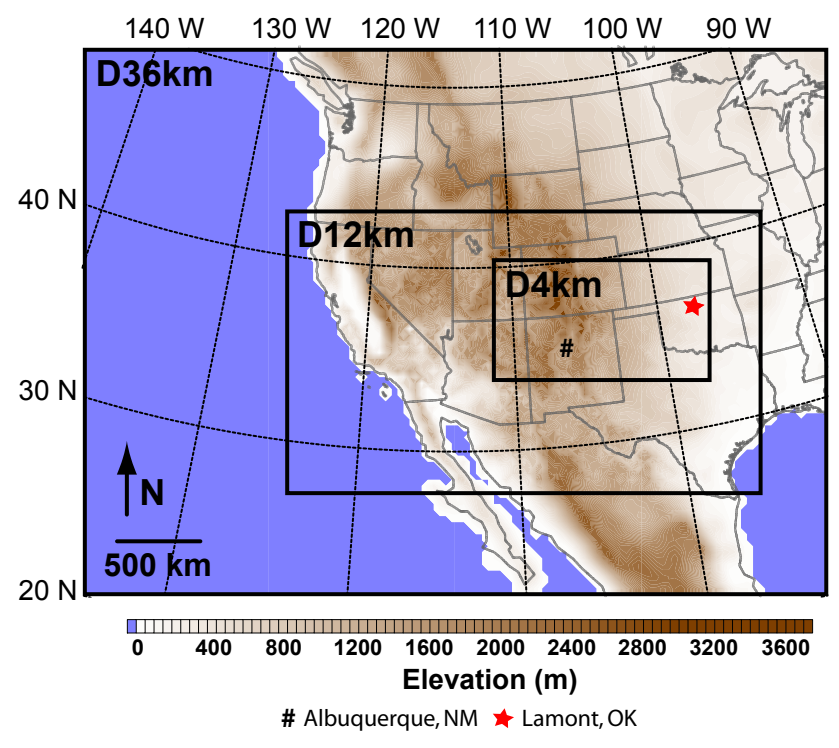

Fig. 3. MM5 domain configuration used for the 19 April 2001 forecast.

Table 1. MM5 V3.7.3 configuration.

\begin{tabular}{lc}
\hline MM5 model specification & Value \\
\hline Forecast duration & $36 \mathrm{~h}$ \\
Forecast start & 12:00 UTC 18 April 2001 \\
Domain spatial resolution & $36 \mathrm{~km}, 12 \mathrm{~km}, 4 \mathrm{~km}$ \\
Domain temporal resolution & $240 \mathrm{~s}, 80 \mathrm{~s}, 27 \mathrm{~s}$ \\
Vertical extent & 50 levels, 0 to $100 \mathrm{~km}$ \\
Vertical resolution & $500 \mathrm{~m}$ from 6 to $13 \mathrm{~km}$ \\
Initialization & NCEP/NCAR Reanalysis \\
Microphysics parameterization & Reisner II \\
Cumulus parameterization & Kain Fritsch \\
Shallow convection option & None \\
Radiation parameterization & CCM2 \\
Nudging & None \\
\hline
\end{tabular}

the Rockies, and a warm front approaching Northern OK (Fig. 4). Circulation vectors with the mean motion of the cold front removed demonstrate that air above $8 \mathrm{~km}$ had net westerly air flow.

Although the MM5 forecast (Fig. 4) was broadly consistent with the National Weather Service (NWS) reanalysis at $850 \mathrm{mb}$ and $500 \mathrm{mb}$, the MM5 had a stronger and tighter Montana low, and a reduced gradient in, and lower overall, $500 \mathrm{mb}$ geopotential heights over the Rockies. In the South Central USA, these model geopotential height biases indicate that the MM5 forecast had lower wind speeds over the Rockies, and weaker frontal lifting than what was observed. 

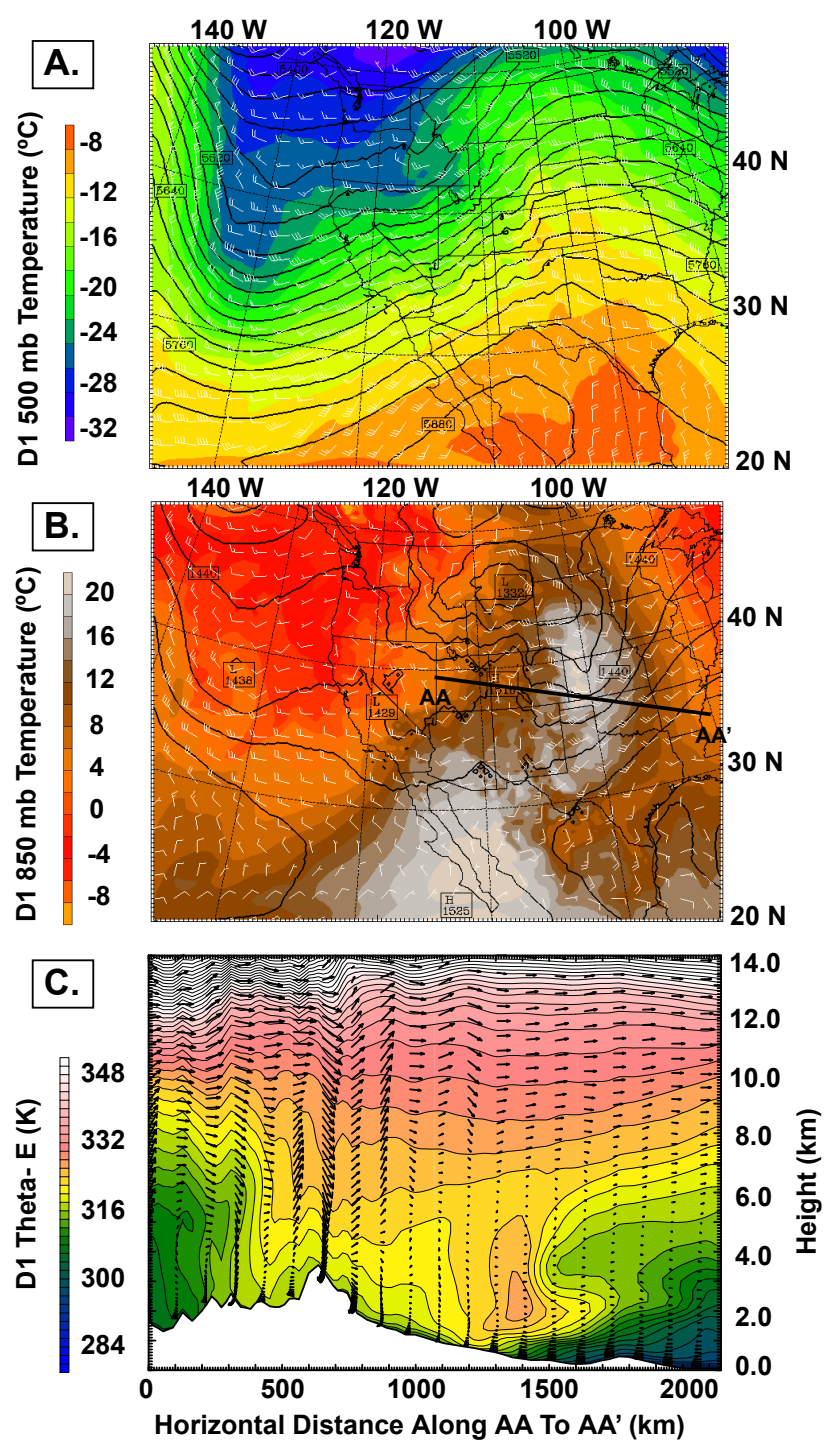

Fig. 4. MM5 meteorology at 12:00 UTC on 19 April 2001 (A) $500 \mathrm{mb}$ temperatures and geopotential heights (B) $850 \mathrm{mb}$ temperatures and geopotential heights. (C) Cross section through AA-AA'. Circulation vectors have the mean speed of the cold front removed $\left(12.7 \mathrm{~m} \mathrm{~s}^{-1}\right)$.

\subsection{MM5 vertical velocities}

Above the Southern Rockies in central New Mexico and Colorado, MM5 forecasted vertically propagating orographic gravity waves with large $\left(w>100 \mathrm{~cm} \mathrm{~s}^{-1}\right)$ and variable $w$ (Fig. 5). The strongest vertical motions resulted from 12:00 to 15:00 UTC when the upper level winds were perpendicular to the Rockies and the cold front aloft approached the Western edge of the lee trough. Downwind of the Rockies, $w$ were generally small $\left(w<30 \mathrm{~cm} \mathrm{~s}^{-1}\right)$.

Using the MM5 wind fields, we calculated Lagrangian $w$ trajectories associated with the observed $\mathrm{Ci}$ formation and evolution (Table 2). Our calculations indicate that air from
Table 2. Lagrangian MM5 trajectories.

\begin{tabular}{lc}
\hline Trajectory parameter & Value \\
\hline MM5 domain & D36km, D4km \\
Duration & $8 \mathrm{~h}$ \\
Temporal resolution & $3.6 \mathrm{~min}$ \\
End time above Lamont & $08: 00$ to $24: 00$ UTC \\
End height above Lamont & $12 \mathrm{~km}$ \\
Total number & 266 \\
\hline
\end{tabular}

Eastern New Mexico and Colorado traveled over the Southern Rockies, and arrived above Lamont in approximately $8 \mathrm{~h}$.

The MM5 domain resolution influenced the amplitude and spatial scale of $w$ variability along the trajectories (Fig. 6). Trajectories derived from the MM5 domain with 4-km spatial resolution $(\mathrm{D} 4 \mathrm{~km})$ had a larger range of $w$ than trajectories derived from the MM5 domain with $36-\mathrm{km}$ spatial resolution $(\mathrm{D} 36 \mathrm{~km})$. The $\mathrm{D} 4 \mathrm{~km}$ trajectories also had greater spectral power at mesoscale frequencies (spatial equivalent $20-60 \mathrm{~km}$ ) than the D36km trajectories. Neither set of trajectories had small-scale variability in $w$ because neither domain resolved dynamics occurring at small scales $\left(v>6 \mathrm{~h}^{-1}\right.$, spatial equivalent $<18 \mathrm{~km}$ ).

The sensitivity of the modeled $w$ to MM5 domain resolution and the lack of $w$ observations made it difficult to quantitatively validate the MM5-forecasted $w$. As a result, we qualitatively assessed the MM5 $w$ forecasts within the context of the two main drivers of orographic wave development: the mountain range topography and the upwind atmospheric stability and wind profile (Durran, 2003).

Mountain wave theory suggests that given the large width of the Front Range, vertically propagating hydrostatic waves should result for most atmospheric stability and wind profiles (Durran, 2003). The D4km $w$ are consistent with this theory (Fig. 5). With the relatively steep leeward slope of the Front Range, idealized calculations suggest hydrostatic gravity waves could generate positive displacement at $\mathrm{Ci}$ heights (see Fig. 20.11 in Durran, 2003). At upper levels, MM5 forecasted persistent positive $w$ at heights of $6-12 \mathrm{~km}$ in the lee of the Rockies (Fig. 5).

To evaluate the representation of the upstream wind and stability profiles, we compared the modeled and observed soundings at Albuquerque, New Mexico (ABQ) (Figs. 7, 3). The MM5 ABQ sounding was qualitatively similar to the observed ABQ sounding. Both soundings had a stable atmospheric potential temperature profile and increasing wind speed with height. Differences between the MM5 and the observed ABQ sounding included: 1) the MM5 sounding was more stable and 2) the MM5 ABQ sounding had less vertical wind speed shear above $8 \mathrm{~km}$. Despite these differences, the MM5 representation of wind and stability profiles provided confidence in the forecasted gravity wave development. 


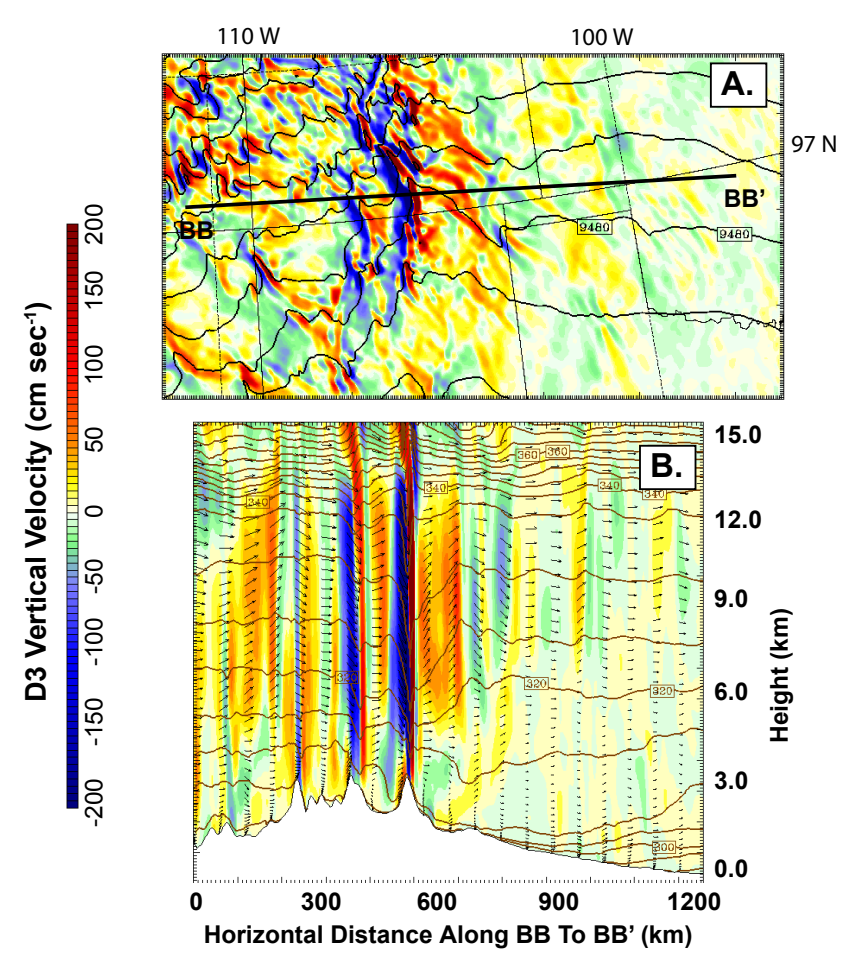

Fig. 5. MM5 vertical velocities at 12:00 UTC on 19 April 2001. (A) D4km $300 \mathrm{mb} w$ (B) D4km $w$ cross section. Location of cross section $\mathrm{BB}^{-\mathrm{BB}^{\prime}}$ is indicated on $(\mathrm{A})$.

\section{Parcel model methods}

\subsection{Conceptual framework}

In this study, we evaluate $\mathrm{Ci}$ processes occurring along Lagrangian $w$ trajectories using the K06 parcel model and a simple conceptual framework (Fig. 8).

The K06 model includes heterogeneous and homogeneous freezing, vapor diffusion, and fallout. Fallout is calculated by assuming that a fraction of the ice particles fall out of the parcel in each time step. This fallout fraction is determined individually for each bin using the particle fall speed, the timestep, and the assumed parcel depth $(H[\mathrm{~m}])$.

The key assumption in our conceptual framework is that processes occurring in an ice formation region near cloud top control $\mathrm{P}(\sigma)$ shape. We model freezing, vapor diffusion, and fallout occurring in this ice formation region with the K06 parcel model. We then calculate $\mathrm{Ci} \sigma$ evolution using Eq. (1) by linearly scaling the ice formation region $R_{\text {eff }}$ and $N_{\text {ice }}$ over the entire cloud depth and by assuming $\Delta \mathrm{z}=3500 \mathrm{~m}$ (the mean observed $\Delta Z$ from 08:00 to 24:00 UTC, see Fig. 2). Finally, we calculate $\mathrm{Ci} \mathrm{P}(\sigma)$ and other distributions such as $\mathrm{P}\left(N_{\text {ice }}\right)$ over the duration of the modeled Lagrangian evolution.

Within our conceptual framework, it is easy to understand and to quantify interactions between complex dynamics and $\mathrm{Ci}$ microphysical processes. In an adiabatic parcel model fol-
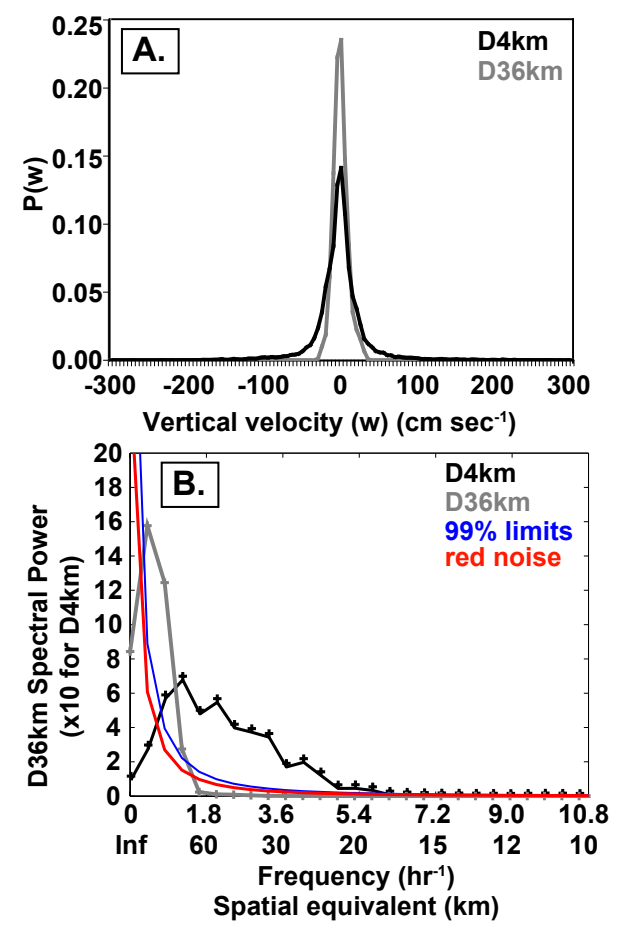

Fig. 6. The effect of MM5 domain resolution on vertical velocity amplitude and frequency structure along Ci Lagrangian evolution pathways. A horizontal wind speed of $30 \mathrm{~m} \mathrm{~s}^{-1}$ was assumed in all spatial equivalence calculations.
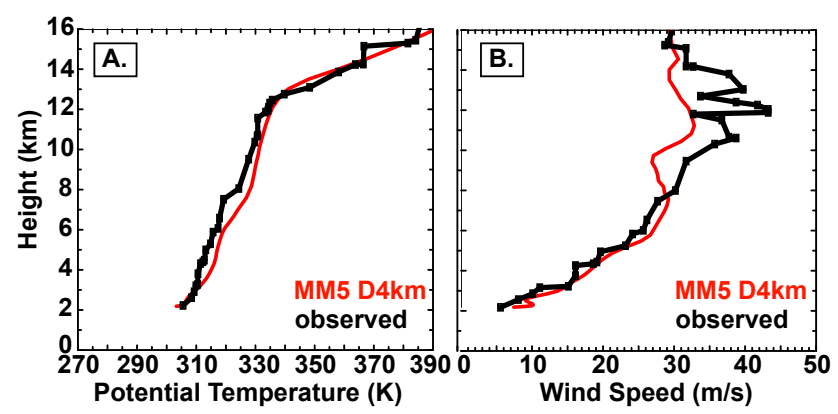

Fig. 7. ABQ 04/19/2001 12 UTC sounding comparison: (A) MM5 D4km vs. observed stability profile (B) MM5 D4km vs. observed wind speed profile.

lowing a Lagrangian displacement trajectory, the time and location of a new freezing event (i.e., when $\frac{d N_{\text {ice }}}{d_{t}}$ increases above a specified threshold) is controlled both by the initial conditions, which set the total displacement required to start freezing, and by the displacement trajectory. If homogeneous freezing begins, the maximum supersaturation with respect to ice largely controls the maximum homogeneous nucleation rate $\left(J_{\text {hom }-\max }\left[\mathrm{m}^{-3} \mathrm{~s}^{-1}\right]\right)$ and the resulting $N_{\text {ice }}$. If heterogeneous freezing begins, $N_{I N}$ and the IN freezing threshold determine the resulting $N_{\text {ice }}$. Once Ci form, their $\sigma$ evolution and $P(\sigma)$ shape are determined by the shortest 
Table 3. Description of parcel model (PM) experiments: All PM experiments are named as follows: PM_MM5Domain_INparameterization(if applicable). All parcels were initialized with sulfuric acid aerosols (dry mass $=10^{-16} \mathrm{~kg}$, $\left.N_{\text {aer }}=100 \mathrm{~cm}^{-3}\right)$. Parcel initial conditions $\left(\mathrm{T}, \mathrm{RH}_{\mathrm{ice}}, \mathrm{P}\right)$ and $w$ trajectories were derived from the indicated MM5 domain. All parcels were assumed to be adiabatic, which implies that no mixing or nudging to the MM5 fields was included. All background IN froze at a shifted water activity equivalent to freezing at $\mathrm{RH}_{\mathrm{ice}}=130 \%$ (see Kärcher and Lohmann, 2003).

\begin{tabular}{lcc}
\hline Experiment name & MM5 domain & IN \\
\hline PM_D4km & D4km & none \\
PM_D4km_IN & D4km & Background IN \\
PM_D4km_Meyers & D4km & Meyers et al. (1992) \\
PM_D36km & D36km & none \\
\hline
\end{tabular}

microphysical and dynamical timescales (see K06). Because the parcel model is a zero-dimensional model, the computational requirements for estimating interactions between realistic dynamics derived from a three-dimensional numerical weather model and binned microphysics are minimal.

Despite the described advantages, there are limitations associated with our methodology for estimating $\mathrm{Ci} \sigma$ evolution (see also K06 and Kay, 2006). For simplicity, we use a constant depth of the ice formation region (the parcel depth $H$ ) and a constant $\Delta z$. Neither of these assumptions is completely realistic. First, $100 \mathrm{~m}$ is a reasonable, but ad hoc, estimate for $H$. Cloud evolution in the K06 model is sensitive to $H$ and vertically resolved cloud processes would be more realistic. Fortunately, K06 found that $\sigma$ trends and $\mathrm{P}(\sigma)$ shapes are largely independent of reasonable $H$ values. Second, using a constant scaling of the formation region properties to obtain an integrated $\mathrm{Ci} \sigma$ cannot always be justified. For example, observations show that $\Delta Z$ variability contributes to $\sigma$ variability (Fig. 2). Because we assume a constant $\Delta Z$, we can only incorporate $\sigma$ variability associated with variability in w and initial conditions such as $N_{I N}$. A more complex model could be used to predict $\Delta Z$ and to assess the contribution of $\Delta Z$ variability to $\sigma$ variability. The fidelity of $\Delta Z$ predictions derived from a more complex model will largely depend on the assumed vertical moisture profile.

\subsection{Parcel model runs}

Guided by the observed Ci cloud top and timing (Fig. 1), we focused our Ci modeling efforts on trajectories ending $12 \mathrm{~km}$ above Lamont (Table 2). We calculated $\mathrm{Ci}$ evolution using the K06 adiabatic parcel model with variable initial conditions and $w$ trajectories (Table 3 ). By comparing $\mathrm{P}(\sigma)$ calculated along $w$ trajectories derived from the $\mathrm{D} 36 \mathrm{~km}$ and $\mathrm{D} 4 \mathrm{~km}$ MM5 domains, we evaluated the influence of mesoscale $w$ variability and $w$ amplitude on Ci formation and evolution.

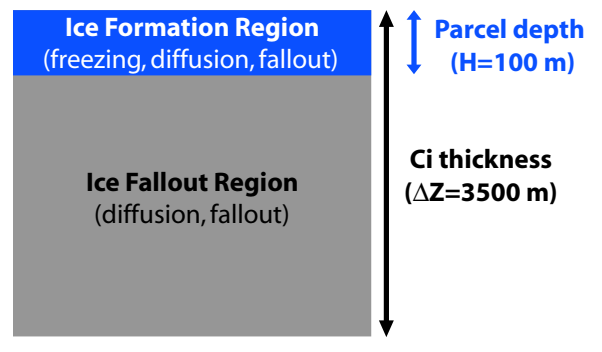

Fig. 8. Ci conceptual framework.

We also evaluated the effect of IN on $\mathrm{Ci} \mathrm{P}(\sigma)$ by initializing parcels with either a fixed background concentration based on observations $\left(N_{I N}=0.03 \mathrm{~cm}^{-3}\right)$ or the $N_{I N}$ predicted by Meyers et al. (1992), a commonly-used model IN parameterization in which the $N_{I N}$ increases exponentially with the vapor supersaturation with respect to ice.

\section{Results}

\subsection{Overview of results}

The Lagrangian dynamical forcing along the MM5 $w$ trajectories revealed large-scale cooling and mesoscale variability in $w$ in the lee of the Rockies. Both the kinematic forcing and the initial conditions affected $\mathrm{Ci}$ processes modeled along the Lagrangian trajectories. Our parcel model results demonstrate that mesoscale $w$ variability associated with orographic gravity waves broadened $\mathrm{Ci} \mathrm{P}(\sigma)$ shape. In contrast, the addition of typical background $N_{I N}\left(N_{I N}=0.03 \mathrm{~cm}^{-3}\right)$ to parcels had a limited influence on modeled $\mathrm{Ci} \sigma$ variability, but did increase overall modeled $\mathrm{Ci}$ cloud cover. Finally, the inhomogeneity, but not the timing, of the observed $\mathrm{Ci}$ was reproduced by our Ci parcel modeling along MM5 trajectories.

\subsection{Lagrangian forcing}

Lagrangian $\mathrm{T}$ and $w$ time-time plots from the D4km MM5 domain show the forcing important for Ci formation and evolution on 19 April 2001 (Fig. 9). All air parcels traveled over the high topography of the Southern Rocky Mountains between evolution times -7 and $-5 \mathrm{~h}$, i.e., 5 to $7 \mathrm{~h}$ before arrival at Lamont. In the lee of the Rocky Mountains, air parcels experienced cooling, and variable $w$ associated with the vertically propagating orographic gravity waves (Fig. 5). For the last $4 \mathrm{~h}$ prior to arrival at Lamont, air parcels had small vertical motions $\left(w<30 \mathrm{~cm} \mathrm{~s}^{-1}\right)$. Widespread cooling occurred between evolution times -3 and $-2 \mathrm{~h}$, while warming occurred along trajectories in the two hours prior to arrival above Lamont. 


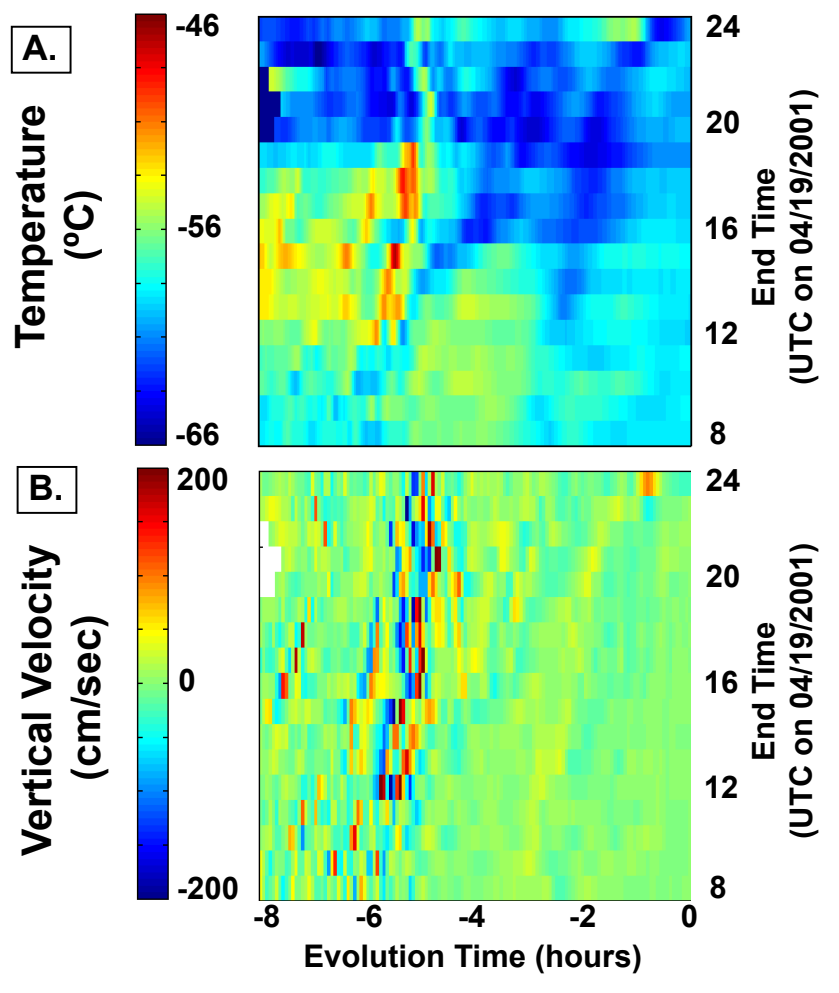

Fig. 9. Temperature and vertical velocity along Lagrangian trajectories derived from the D4km MM5 domain: These time-time plots show the Lagrangian evolution of the MM5 kinematic forcing on air parcels arriving $12 \mathrm{~km}$ above Lamont. The y-axis indicates the parcel arrival time at Lamont. The $\mathrm{x}$-axis indicates the evolution time, i.e., the time before parcel arrival at Lamont.

\subsection{Parcel model Ci}

Consistent with observations (Fig. 1), cooling in the lee of the Rockies resulted in parcel model $\mathrm{Ci}$ formation from 06:00 to 16:00 UTC, i.e., between evolution times -6 and $-4 \mathrm{~h}$ for trajectories arriving above Lamont from 12:00 to 20:00 UTC (Fig. 10). Variability in the large-scale cooling, the mesoscale $w$ amplitude and timing, and the initial conditions resulted in a range of parcel modeled $\mathrm{Ci}$ formation times, $N_{\text {ice }}, \sigma$, and cloud lifetimes along the Lagrangian trajectories. From the parcel model $\mathrm{Ci}$ results, three general $\mathrm{Ci}$ formation and evolution sequences could be categorized by arrival time at Lamont:

1. Along trajectories arriving from $12: 00$ to $14: 00$ UTC, $\mathrm{Ci}$ formed by homogeneous freezing at evolution time $-5 \mathrm{~h}$, but $\mathrm{Ci}$ then sublimated in descending motions. After a cloud-free period, a second homogeneous freezing event occurred at evolution time $-3 \mathrm{~h}$ and these $\mathrm{Ci}$ persisted to Lamont.

2. Along trajectories arriving from 15:00 to 20:00 UTC, $\mathrm{Ci}$ formed from evolution time -5 to $-3 \mathrm{~h}$. Variability in $w$ led to a range of $N_{\text {ice }}$ and cloud lifetimes. Only the tra-
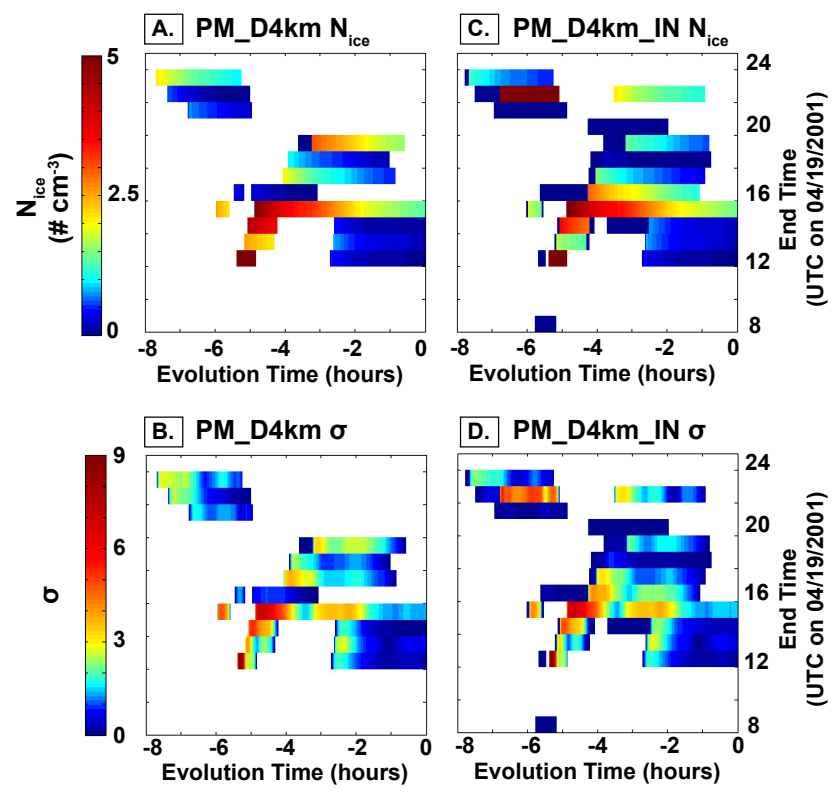

Fig. 10. Parcel model Ci along hourly trajectories: $N_{\text {ice }}$ and $\sigma$ from the parcel model are plotted along Lagrangian trajectories ending every hour $12 \mathrm{~km}$ above Lamont. White indicates no cloud was present (IWC $<0.01 \mathrm{mg} \mathrm{m}^{-3}$ ). See Table 3 for parcel model configuration details and naming conventions. See Fig. 9 for a description of time-time plots.

jectories with large $N_{\text {ice }}$ and limited descending motion persisted over many hours and arrived at Lamont.

3. Along trajectories arriving from 21:00 to $24: 00$ UTC, $\mathrm{Ci}$ formed from evolution time -8 to $-7 \mathrm{~h}$, but few $\mathrm{Ci}$ formed in the lee of the Rockies, and no $\mathrm{Ci}$ arrived at Lamont.

The addition of background IN to air parcels resulted in changes to the timing and magnitude of homogeneous freezing along individual trajectories; however, the overall location of cloud formation in the lee of the Rockies, the variability in $N_{\text {ice }}$ and cloud lifetimes, and the quantity of $\mathrm{Ci}$ arriving at Lamont were not altered by the addition of background IN (Fig. 10).

Both $N_{\text {ice }}$ variability and cloud lifetime variability were robust features of the parcel model $\mathrm{Ci}$. In contrast, the Reisner II Ci had limited variability in $N_{\text {ice }}$ and Ci cloud evolution (Fig. 11). These differences suggest that while the largescale forcing controlled the location of $\mathrm{Ci}$ formation, homogeneous freezing at locally variable $w$ produced the modeled $N_{\text {ice }}$ and Ci evolution variability.

Because changes along individual or even hourly trajectories are not necessarily representative of overall changes, we statistically assessed the influence of mesoscale $w$ variability and $N_{I N}$ specification on parcel model Ci by comparing $\mathrm{P}(\sigma)$ and $\mathrm{P}\left(N_{\text {ice }}\right)$ calculated along all 266 Lagrangian trajectories. 


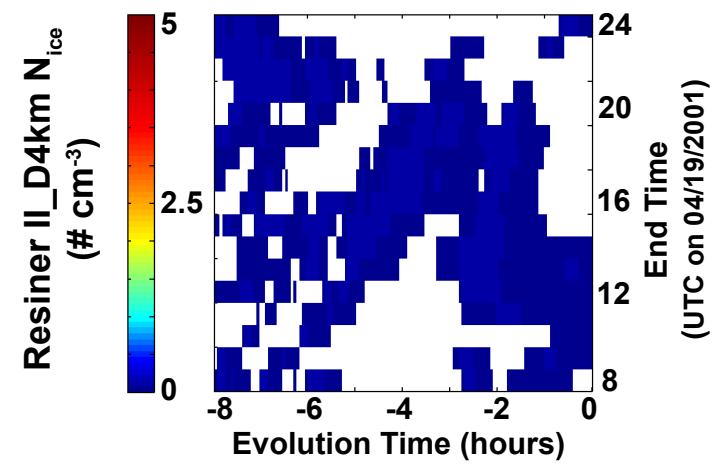

Fig. 11. Reisner II Ci along hourly trajectories: $N_{\text {ice }}$ generated by the Reisner II microphysical scheme in MM5 D4km are plotted along trajectories ending $12 \mathrm{~km}$ above Lamont. White indicates no cloud was present (IWC $<0.01 \mathrm{mg} \mathrm{m}^{-3}$ ). See Fig. 9 for a description of time-time plots.

Comparison between $\mathrm{P}(\sigma)$ and $\mathrm{P}\left(N_{\text {ice }}\right)$ derived along all of the D36km and D4km trajectories illustrates the influence of mesoscale $w$ variability and $w$ amplitude on Ci properties (Fig. 12). Along D4km trajectories, large $w$ associated with mesoscale $w$ variability broadened $\mathrm{P}(\sigma)$ and shifted $\mathrm{P}\left(N_{\text {ice }}\right)$ towards large values. Given that large $N_{\text {ice }}$ lead to long $\tau_{\text {fallout }}$ and long cloud lifetimes, we expected more cloud cover along the D4km trajectories than along the D36km trajectories. We found the opposite: D4km trajectories had 3\% less cloud cover than D36km trajectories. Owing to their differing spatial resolutions, the D36km and D4km domains had different representations of the large-scale cooling and gravity wave evolution in the lee of the Rockies. Indeed, both the amplitude and the frequency structure of Lagrangian $w$ varied with the spatial resolution of the MM5 domain (Fig. 6). In the end, our analysis could not isolate the influence of mesoscale $w$ variability on cloud lifetimes and cloud cover from these systematic differences related to the spatial resolution of the resolved dynamics.

Adding $N_{I N}$ to parcels increased $\mathrm{Ci}$ cloud cover along the Lagrangian trajectories from $25 \%\left(N_{I N}=0\right)$ to $30 \%$ for background $N_{I N}$ and to $32 \%$ for Meyers et al. (1992) $N_{I N}$ (Fig. 12). This cloud cover increase occurred only for optically thin $\mathrm{Ci}$ and resulted because heterogeneous freezing occurred at a lower $\mathrm{RH}_{\text {ice }}$ than homogeneous freezing (see Table 3).

The impact of IN on optically thick Ci depended on $N_{I N}$. Due to scavenging and their relatively low concentrations, background IN had little impact on the large $\sigma$ and large $N_{\text {ice }} \mathrm{Ci}$ that formed by homogeneous freezing. In contrast, the use of the Meyers et al. (1992) parameterization resulted in a large addition of $N_{I N}$ to parcels and a decrease in the occurrence of large $N_{\text {ice }}$ and large $\sigma \mathrm{Ci}$. The Meyers et al. (1992) parameterization produced more IN than are typically observed in the upper troposphere (Meyers $N_{I N}>0.3 \mathrm{~cm}^{-3}$ ). Therefore, we suggest that our background $N_{I N}$ modeling results are more likely to represent the typical influence of IN on Ci.

\subsection{Comparison of modeled and observed $\mathrm{Ci}$}

Both the parcel model and Reisner II scheme reproduced the observed orographic Ci formation in the lee of the Rockies; however, both models failed to reproduce the observed $\mathrm{Ci}$ presence above Lamont. The lidar depolarization showed $\mathrm{Ci}$ occurring continuously from 08:00 to 24:00 UTC (Fig. 1), yet both the parcel model (Fig. 10) and Reisner II (Fig. 11) produced no Ci above Lamont after 16:00 UTC.

Differences between modeled and observed horizontal advection may have contributed to a lack of modeled Ci presence above Lamont. At a height of $12 \mathrm{~km}$, the MM5 horizontal wind speeds were up to $10 \mathrm{~m} \mathrm{~s}^{-1}$ smaller than observed horizontal wind speeds (Fig. 7). For a fixed Ci cloud lifetime, increasing the horizontal wind speed could alter orographic Ci presence above Lamont. For example, an increase in model advection speeds may have allowed parcel model $\mathrm{Ci}$ to persist farther from the Rockies and to arrive above Lamont from 16:00 to 20:00 UTC (Fig. 10). Yet, horizontal advection speed cannot entirely explain differences between observed and modeled Ci presence. The GOES observations indicate that Ci persisted after passing above Lamont (Fig. 1).

A moisture deficit could explain the differences between the modeled and observed $\mathrm{Ci}$ presence. Modeled $\mathrm{RH}_{\text {ice }}$ above Lamont were $<100 \%$ after 15:00 UTC, which promoted sublimation of the orographic $\mathrm{Ci}$ and inhibited new freezing events (Fig. 13). Low $\mathrm{RH}_{\text {ice }}$ resulted from net subsidence (warming) in the two hours before trajectories arrived at Lamont (Fig. 9). The model moisture deficit could have resulted because the MM5 forecast did not adequately capture warm frontal lifting throughout the South Central USA. The reduced southern extent of Montana low in the MM5 forecast as compared to the NWS reanalysis supports this hypothesis.

Given modeled $\mathrm{RH}_{\text {ice }}<100 \%$, it is not surprising that few model $\mathrm{Ci}$ arrived at Lamont. With modeled $\mathrm{RH}_{\mathrm{ice}}>100 \%$, more orographic $\mathrm{Ci}$ may have survived and arrived at Lamont. In addition, only modest lifting is required for parcels near ice saturation to reach a homogeneous freezing threshold. If model parcels were lifted an additional 300 to $400 \mathrm{~m}$, new homogeneous freezing events could have occurred and $w$ variability could have resulted in variable $N_{\text {ice }}$.

Despite obvious differences between the observed and modeled $\mathrm{Ci}$ presence, model $\mathrm{Ci}$ did form in the lee of the Rockies and were advected to Lamont (Figs. 10, 11). By comparing the observed and modeled Ci properties (Table 4), we found that the parcel model helped explain the $\mathrm{Ci}$ observations in the following ways:

1. Observed $\Delta Z$ variability clearly contributed to the observed broad $\mathrm{P}(\sigma)$ (Fig. 2); however, the observed broad $\mathrm{P}(\sigma)$ at Lamont (Fig. 2) could also be partially explained by variable $N_{\text {ice }}$ resulting from homogeneous freezing 

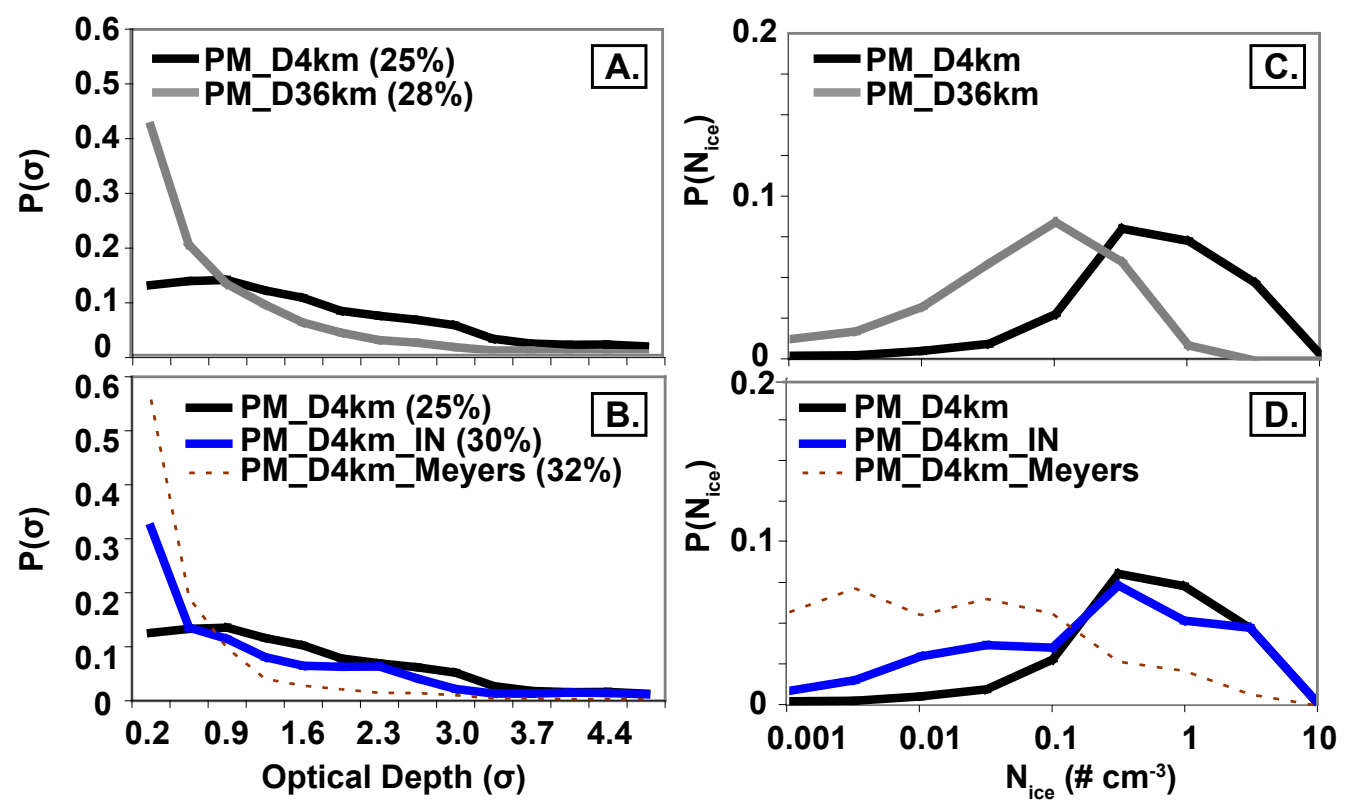

Fig. 12. Influence of MM5 domain resolution and IN parameterization on parcel model $\mathrm{P}(\sigma)$ and $\mathrm{P}\left(N_{\text {ice }}\right) . \mathrm{P}(\sigma)$ and $\mathrm{P}\left(N_{\text {ice }}\right)$ were calculated along trajectories ending $12 \mathrm{~km}$ above Lamont from 12:00 to 24:00 UTC. The cloud fraction (cf) is listed after the parcel model experiment name. Cloudy air must have an IWC $>0.01 \mathrm{mg} \mathrm{m}^{-3}$. $\mathrm{P}\left(N_{\text {ice }}\right)$ were calculated for $N_{\text {ice }}>0.001 \mathrm{~cm}^{-3}$. $\mathrm{P}(\sigma)$ were calculated for $\sigma>0.1$. The Meyers $\mathrm{P}(\sigma)$ and $\mathrm{P}\left(N_{\text {ice }}\right)$ are dashed because the Meyers et al. (1992) parameterization resulted in $N_{I N}$ that are not atmospherically relevant. See Table 3 for parcel model experiment details and naming conventions.

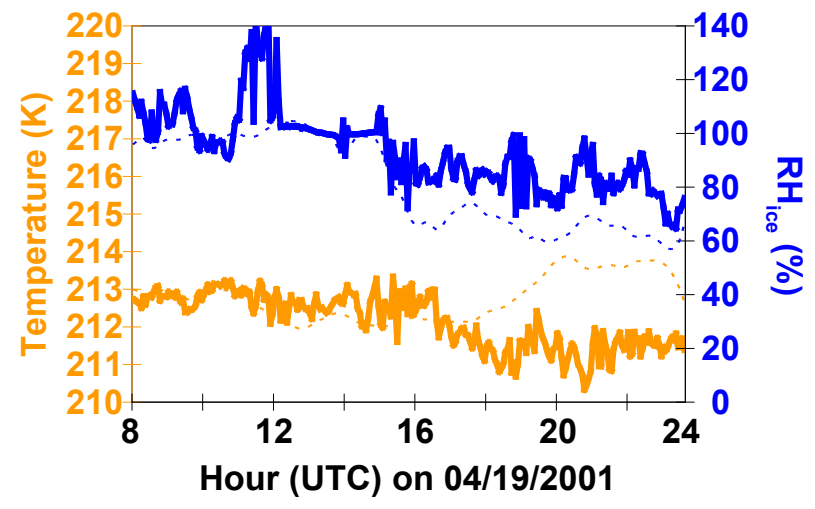

Fig. 13. Model humidity and temperature $12 \mathrm{~km}$ above Lamont: Both the parcel model (PM_D4km, solid lines) and the Reisner II scheme (ReisnerII_ D4km, dotted lines) air at Ci levels above Lamont was sub-saturated with respect to ice.

at variable $w$. Although the modeled $\mathrm{P}(\sigma)$ (Fig. 12) are not coincident in time and space with the $\mathrm{P}(\sigma)$ observations at Lamont, broad modeled $\mathrm{P}(\sigma)$ along trajectories resulted from homogeneous freezing occurring at variable $w$.

2. Large $N_{\text {ice }}$ resulted in long parcel modeled Ci lifetimes. Thus, the parcel model could help explain the observed persistence of Ci over many hours in the GOES imagery.
Table 4. Model vs. observed variability in $\mathrm{Ci}$ properties above Lamont from 08:00 to 24:00 UTC on 19 April 2001. Model values are only included for model IWC $>0.01 \mathrm{~m} \mathrm{~m}^{-3}$.

\begin{tabular}{lccc}
\hline Source & $\begin{array}{c}N_{\text {ice }} \\
\mathrm{cm}^{-3}\end{array}$ & $\begin{array}{c}R_{\text {eff }} \\
\mu \mathrm{m}\end{array}$ & $\begin{array}{c}\sigma \\
\text { dimensionless }\end{array}$ \\
\hline PM_D4km & $0.01-3.05$ & $1-25$ & $0.01-2.3$ \\
PM_D4km _IN & $0.01-2.71$ & $6-30$ & $0.01-2.3$ \\
PM_D4km_Meyers & $0.001-1.04$ & $5-25$ & $0.01-1.1$ \\
ReisnerII_D4km & $0.02-0.08$ & $25-36$ & NA \\
Observed & NA & NA & $0-3$ \\
\hline
\end{tabular}

\section{Conclusions}

Using self-consistent Lagrangian trajectories derived from a mesoscale weather model and an adiabatic parcel model with binned ice microphysics, this study evaluated the influence of mesoscale $w$ and IN presence on Ci $N_{\text {ice }}$, and inhomogeneity during an orographic $\mathrm{Ci}$ case study. The primary findings were:

- When mesoscale variability (along-path fluctuations with timescales of $\left.2 \mathrm{~h}^{-1}<v<10 \mathrm{~h}^{-1}\right)$ in $w$ affected homogeneous freezing, $\mathrm{P}(\sigma)$ derived along Lagrangian trajectories were broad. Broad $\mathrm{P}(\sigma)$ resulted because homogeneous freezing driven by variable $w$ led to variable $N_{\text {ice }}$, variable $\sigma$, and variable Ci lifetimes. 
- The addition of IN to air parcels increased cloud cover along Lagrangian trajectories by 5 to $7 \%$, depending on the $N_{I N}$ and IN freezing threshold. Whereas background $N_{I N}\left(N_{I N}=0.03 \mathrm{~cm}^{-3}\right)$ presence had little influence on the occurrence of large $\sigma$, the presence of large $N_{I N}\left(N_{I N}>0.3 \mathrm{~cm}^{-3}\right)$, resulting from use of Meyers et al. (1992) parameterization, decreased the occurrence of large $\sigma$ by suppressing homogeneous freezing. Because the Meyers et al. (1992) parameterization produced more IN than are typically observed in the upper troposphere $\left(N_{I N}<0.1 \mathrm{~cm}^{-3}\right.$ (DeMott et al., 2003; Rogers et al., 1998)), the background $N_{I N}$ modeling results are representative of what occurs in the atmosphere.

- All models predicted fewer Ci than were observed. Low humidities along modeled trajectories, which were attributed to a lack of MM5 frontal lifting, could explain differences in modeled and observed $\mathrm{Ci}$. Nevertheless, the parcel model $\mathrm{Ci}$ helped explain observed $\mathrm{Ci}$ inhomogeneity in the following sense: 1) Broad observed $\mathrm{P}(\sigma)$ could be partially explained by variable $N_{\text {ice }}$ arriving along parcel model Ci trajectories, 2) Large $N_{\text {ice }}$ predicted by the parcel model resulted in long $\mathrm{Ci}$ lifetimes and could explain the persistence of Ci over many hours.

Although there are limitations associated with using an adiabatic parcel model and trajectories to represent $\mathrm{Ci}$ processes and properties, the results from this study demonstrate clear connections between mesoscale $w, \mathrm{Ci} N_{\text {ice }}$, and $\mathrm{Ci} \mathrm{P}(\sigma)$. Our results support and extend the results of Kärcher and Ström (2003); Hoyle et al. (2005); Haag and Kärcher (2004), who suggested that $w$ variability and homogeneous freezing generate $N_{\text {ice }}$ variability in the atmosphere.

The primary goal of this study was to illustrate the influence of mesoscale $w$ and $N_{I N}$ variability on $\mathrm{Ci} \mathrm{P}(\sigma)$ along numerous realistic Lagrangian trajectories. Therefore, we were not alarmed to find deviations between modeled and observed Ci presence. A mesoscale model forecast is an initial value problem with a single realization. We could have generated MM5 forecasts until we reproduced the observed Ci presence, but a detailed reproduction of the observations was not our goal. The observations were invaluable because they helped us identify 19 April 2001 as a good case study, not because they provided a benchmark for evaluating the ability of models to exactly reproduce observations.

Given the limitations of this study, and that this is only a single case study, the influence of $w$ and IN on Ci cloud properties should be explored further. In particular, modeling $\mathrm{Ci}$ evolution along trajectories derived from models that resolve $w$ variability at small spatial scales $\left(v>6 \mathrm{~h}^{-1}\right)$ would be useful. In addition, including the effects of variable $\Delta \mathrm{z}$ on $\mathrm{Ci}$ $\mathrm{P}(\sigma)$ and comparing the influence of $\Delta \mathrm{z}$ with the influence of $w$ highlighted by this study would be interesting. It is important to note that using a more complex model to assess the influence of $\Delta Z$ variability on $\sigma$ variability requires an accurate vertical moisture profile. Finally, we recommend investigation of the parallels between the $w-N_{\text {ice }}$-cloud lifetimecloud cover connections described in this study and the indirect effects of aerosols on stratus albedos, lifetimes, and cloud cover (e.g. Twomey, 1974; Albrecht, 1989).

Acknowledgements. We acknowledge NSF-ATM-02-1147 for research funding and the NCAR Scientific Computing Division for providing computing resources. J. E. Kay was supported in part by the Office of Biological and Environmental Research of the U.S. Department of Energy under contract DE-AC06-76RL01830 to the Pacific Northwest National Laboratory as part of the Atmospheric Radiation Measurement Program. The Pacific Northwest National Laboratory is operated by Battelle for the U.S. Department of Energy. We thank T. Ackerman, Q. Fu, and J. Locatelli for productive scientific discussions, D. Turner for providing and helping to interpret the Raman lidar observations, and M. Stoelinga and D. Durran for help with the MM5 simulations.

Edited by: B. Kärcher

\section{References}

Albrecht, B.: Aerosols, cloud microphysics and fractional cloudiness, Science, 245, 1227-1330, 1989.

Carlin, B., Fu, Q. Lohmann, U., Mace, G., Sassen, K., and Comstock, J.: High-cloud horizontal inhomogeneity and solar albedo bias, J. Clim., 15(17), 2321-2339, 2002.

Dean, S. M., Lawrence, B. N., Grainger, R. G. and Heuff, D. N.: Orographic cloud in GCM: the missing cirrus, Clim. Dyn., 24, 771-780, 2005.

DeMott, P. J., Cziczo, D. J., Prenni, A. J., Murphy, D. M., Kreidenweis, S. M., Thomson, D. S., Borys, R., and Rogers, D. C.: Measurements of the concentration and composition of nuclei for cirrus formation, Proc. Natl. Acad. Sci., 100(25), 14 655-14 660, 2003.

Durran, D. R.: Lee Waves and Mountain Waves, in: Encyclopedia of Atmospheric Science, edited by: Holton, J., Pyle, J., and Curry, J., Academic Press, 2003.

Field, P. R., Cotton, R. J., Noone, K., Glantz, P., Kaye, P. H., Hirst, E., Greenaway, R. S., Jost, C., Gabriel, R., Reiner, T., Andreae, M., Saunders, C. P. R., Archer, A., Choularton, T., Smith, M., Brooks, B., Hoell, C., Bandy, B., Johnson, D., and Heymsfield, A.: Ice nucleation in orographic wave clouds: Measurements made during INTACC, Q. J. Roy. Meteor. Soc., 127(575), 14931512, 2001.

Fu, Q., Carlin, B., and Mace, G.: Cirrus horizontal inhomogeneity and OLR bias, Geophys. Res. Lett., 27(20), 3341-3344, 2000.

Grell, G. A., Dudhia, H., and Stauffer, D. R.: A description of the fifth generation Penn State NCAR Mesoscale Model (MM5), NCAR Tech Note NCAR/TN-398+STR, National Center for Atmospheric Research (NCAR), Boulder, CO, 121 pp., 1994.

Haag, W. and Kärcher, B.: The impact of aerosols and gravity waves on cirrus clouds at mid-latitudes, J. Geophys. Res., 109, D12202, doi:10.1029/2004JD004579, 2004.

Heymsfield, A. J. and Miloshevich, L.: Relative Humidity and Temperature Influences on Cirrus Formation and Evolution: Obser- 
vations from Wave Clouds and FIRE II, J. Atmos. Sci., 52, 43024326, 1995.

Hoyle, C. R., Luo, B. P., and Peter, T.: The origin of high ice crystal number densities in cirrus clouds, J. Atmos. Sci., 62, 2568-2579, 2005.

Kärcher, B. and Lohmann, U.: A parameterization of cirrus cloud formation: heterogeneous freezing, J. Geophys. Res., 108, D14, doi:10.1029/2002JD003220, 2003.

Kärcher, B. and Ström, J.: The roles of dynamical variability and aerosols in cirrus cloud formation, Atmos. Chem. Phys., 3, 823838,2003 ,

http://www.atmos-chem-phys.net/3/823/2003/.

Kay, J. E.: Physical controls on cirrus cloud inhomogeneity, Ph.D. Thesis, University of Washington, 2006.

Kay, J. E., Baker, M., and Hegg, D.: Microphysical and dynamical controls on cirrus cloud optical depth distributions, J. Geophys. Res., 111, D24205, doi:10.1029/2005JD006916, 2006.

Koop, T., Luo, B., Tslas, A., and Peter, T.: Water activity as the determinant for homogeneous ice nucleation in aqueous solutions, Nature, 406, 611-614, 2000.
Mace, G. G., Clothiaux, E. E., and Ackerman, T. P.: The composite characteristics of cirrus clouds: bulk properties revealed by one year of continuous cloud radar data, J. Climate, 14, 2185-2203, 2001.

Meyers, M. P., DeMott, P. J., and Cotton, W. R.: New primary icenucleation parameterization in an explicit cloud model, J. Appl. Meteorol., 55, 2039-2052, 1992.

Reisner, J., Rasmussne, R. M., and Bruintjes, R. T.: Explicit forecasting of supercooled liquid water in winter storms using the MM5 mesoscale model, Q. J. Roy. Meteor. Soc., 124,1071-1107, 1998.

Rogers, D. C., DeMott, P. J., Kredenweis, S. M., and Chen, Y.: Measurements of ice nucleating aerosols during SUCCESS, Geophys. Res. Lett., 25(9), 1383-1386, 1998.

Turner, D. D.: Arctic mixed-phase cloud properties from AERI lidar observations: Algorithm and results from SHEBA, J. Appl. Meteorol., 44(4), 427-444, 2005.

Twomey, S. A.: Pollution and the planetary albedo, Atmos. Environ., 8, 1251-1256, 1974. 